\title{
Saúde Coletiva e Educação Física: distanciamentos e interfaces
}

\author{
Public Health and Physical Education: divergences and interfaces
}

Júlia Aparecida Devidé Nogueira ${ }^{1}$

Maria Lúcia Magalhães Bosi ${ }^{2}$

${ }^{1}$ Programa de Pós-

Graduação em Educação

Física, Faculdade

de Educação Física,

Universidade de Brasília.

Campus Universitário Darcy

Ribeiro. 71000-000 Brasília

DF Brasil.

julianogueira@unb.br

${ }^{2}$ Programa de Pós-

Graduação em Saúde

Coletiva, Departamento

de Saúde Comunitária,

Faculdade de Medicina,

Universidade Federal do

Ceará. Fortaleza CE Brasil.
Abstract The article examines divergences and interfaces between the fields of Physical Education (PE) and Public Health (PH) in the Brazilian context, highlighting tensions in the supremacy for scientific authority and competence in line with the concepts of 'scientific field' and 'nuclei of knowledge'. Understanding PH as an institutionalized, legitimized and scientifically consolidated field with three fundamental nuclei: Epidemiology; Social and Human Sciences; and Policy, Planning and Management; we show its approximation to the PE field, resulting in specific knowledge and practices of $P E$ in $P H$. By stressing risks of a sedentary lifestyle, Epidemiology was the first nucleus of linkage to this new field. Nevertheless, reflections from the Social Sciences broaden the understanding of the PE/PH objects of study by recognizing the multidimensional nature of the corporal culture related to health. However, the Policies nucleus only took shape when physical activity became a priority in a set of policies and programs to combat chronic diseases and promote health. Tensions between the biological and social paradigms define the configuration of the fields and represent a major challenge to be overcome by $\mathrm{PE} / \mathrm{PH}$, albeit the critical benchmark in health offers a promising way for overcoming it.

Key words Scientific field, Nuclei of knowledge, Public health, Physical education
Resumo O artigo examina distanciamentos e interfaces entre os campos da Educação Física (EF) e da Saúde Coletiva (SC) no contexto brasileiro, explicitando tensionamentos na luta pela autoridade e competência científicas, consoante os conceitos de "campo cientifico" e "núcleos de saberes". Compreendendo a SC como campo institucionalizado, legitimado e cientificamente consolidado com três núcleos fundamentais: Epidemiologia; Ciências Sociais e Humanas; e Políticas, Planejamento e Gestão; evidenciamos sua aproximação com o campo da EF, resultando em saberes e práticas específicas da EF em SC. Ao naturalizar a linguagem do risco do sedentarismo, a Epidemiologia foi o primeiro núcleo de vinculação desse novo campo. Não obstante, reflexões vindas das Ciências Sociais ampliam a visão dos objetos de estudo da EFSC ao reconhecer a natureza multidimensional da cultura corporal vinculada à saúde. Já o núcleo Políticas só adquire contornos recentemente, quando a atividade física se torna prioridade no conjunto de politicas e programas de combate às doenças crônicas e de promoção da saúde. Tensões entre os paradigmas biológico e social marcam a conformação dos campos e representam um desafio a ser vencido pela EFSC, mas o referencial crítico em saúde oferece um caminho promissor para esta superação.

Palavras-chave Campo científico, Núcleos de saberes, Saúde coletiva, Educação Física 


\section{Introdução}

O presente artigo busca examinar a configuração epistemológica de um importante núcleo de saberes e práticas: a Educação Física em Saúde Coletiva (EFSC), que emerge e vem se consolidando no contexto sanitário brasileiro, em compasso com fenômenos como as transições nutricional e demográfica e o destaque crescente dado à atividade física nos protocolos em saúde, tanto na dimensão coletiva como individual ${ }^{1-3}$. Não obstante essa relevância, análises sobre essa configuração constituem um tema ainda lacunar na literatura cientifica. Trata-se de um espaço que vem sendo construído a partir da interface de seus campos de origem, a Saúde Coletiva (SC) e a Educação Física (EF), ambos reconhecidos como domínios específicos, multidimensionais e marcados pela interdisciplinaridade, nos quais convivem, de forma por vezes conflitiva, distintos saberes e paradigmas $^{4,5}$. No Brasil, cada um desses campos possui redes de formação que guardam mútua autonomia, cabendo destacar a recente emergência de cursos de graduação em $\mathrm{SC}^{6}$ e a divisão da graduação em EF em licenciatura e bacharelado ${ }^{7}$.

A EFSC ocupa um espaço de interseção que mantém características próprias dos campos originários, mas também evidencia algumas rupturas com seus contornos seminais. Para examinar esse novo âmbito de saberes e práticas que vem se configurando no Brasil optamos por revisitar, de início, os domínios da SC e da EF, buscando identificar os saberes que os constituem, os movimentos entre eles, e as interações que se processam entre os campos, de modo a, na sequência, evidenciar algumas aproximações, distanciamentos e interfaces da EFSC sem, contudo, ter a pretensão de esgotar todas as dimensões dessa complexa análise no presente artigo.

Para auxiliar a identificação dos objetos, conceitos e métodos que marcam cada um dos campos específicos utilizamos o constructo "núcleos de saberes" correspondendo aos espaços e formações disciplinares de cada campo ${ }^{4}$, tal como proposto por Bosi e Prado ${ }^{8}$ em análise concernente ao campo da Alimentação e Nutrição em Saúde Coletiva, cujo modelo inspira este artigo. Tratase, portanto, de um exercício reflexivo-analítico, orientado por um arcabouço conceitual mediante o qual analisamos configurações epistemológicas de dois campos e a dinâmica entre os mesmos. Nessa análise, consideramos também as relações de poder que perpassam as estruturas de pensamento partilhadas por uma comunidade, sem o que não poderíamos compreender fenômenos como a emergência e a hegemonia conquistada por cada paradigma em dado momento históri$\mathrm{CO}^{9}$. Reconhecemos aproximações e contradições que perpassam a constituição de cada um dos campos de origem, buscando explicitar dois planos de tensionamento: um de ordem epistemológica e outro, de natureza político-ideológica. Ao considerar os embates epistemológicos como tensões intrinsecamente políticas entre estruturas conceituais operantes tanto no plano conceitual quanto na práxis do campo ${ }^{9}$ adotamos o conceito de "campo científico", entendido como espaço de luta concorrencial em torno de monopólios da autoridade e da competência científicas ${ }^{10}$.

\section{O campo da Saúde Coletiva}

A constituição da Medicina Social, da Saúde Pública e da SC vinculam-se estreitamente às políticas de saúde e expressam especificidades do contexto sócio-histórico em que emergiram. A Medicina Social tem origem associada aos projetos conhecidos como polícia médica na Alemanha, medicina urbana na França e medicina da força de trabalho na Inglaterra. A Saúde Pública é um projeto que se vincula à emergência do capitalismo na Europa e seus efeitos na saúde como desdobramentos da industrialização, urbanização desordenada e aumento da miséria relativa. Já o campo da SC surge no Brasil a partir de 1970, quando uma vertente crítica no interior da Saúde Pública oferece resistência à posição dominante das teorias lineares da causalidade e busca conjunções interdisciplinares e críticas, visando a superar, dialeticamente, o modelo biomédico ${ }^{4}$.

Atualmente, o acúmulo de reflexões sobre o campo da SC nos permite declará-lo como espaço institucionalizado, legitimado e cientificamente consolidado, apresentando uma natureza epistemológica e uma prática política diferenciadas fundadas na interface das Ciências Naturais e das Ciências Humanas e Sociais ${ }^{11}$. O campo da $\mathrm{SC}$ se fundamenta a partir de três núcleos de saberes principais: a Epidemiologia; (a parcela das) Ciências Humanas e Sociais que transita nesse campo; e o domínio que, sob múltiplos rótulos, se ocupa da Política, do Planejamento elou da Planificação e da Gestão de Sistemas de Saúde ${ }^{8}$.

O núcleo da Epidemiologia tem por objeto "a distribuição e os determinantes dos processos de saúde e doença em populações humanas" ${ }^{\prime 2}$. Em compasso com as tendências dominantes da ciência moderna, tem-se a evidente hegemonia da Epidemiologia sobre os demais núcleos no âmbito da SC, notadamente sobre as humanidades ${ }^{8}$. 
Não obstante, no plano epistemológico, a SC passa a apresentar interseções cada vez mais amplas e profundas com o núcleo das Ciências $\mathrm{Hu}$ manas e Sociais em Saúde visando a obter a transdisciplinaridade exigida pelos objetos de que se ocupa $^{13}$. As teorias e metodologias consolidadas a partir desse núcleo foram indispensáveis para o desenvolvimento e a consolidação do campo ${ }^{14}$. Ao lidar com dimensões qualitativas e quantitativas; sujeito e estrutura; natureza e história; objetividade e subjetividade ${ }^{8}$, representaram a base da inovação e da práxis em $\mathrm{SC}^{4}$. A contribuição da teoria crítica nas análises desenvolvidas permite então repensar o método e o processo de construção de enunciados científicos, não apenas mensurando, mas contextualizando socialmente o processo saúde-doença.

As Ciências Humanas e Sociais ajudam também a reconfigurar o núcleo da Epidemiologia, inserindo elementos conceituais e metodologias que a dotam de uma visão crítica ou social, e fornecem bases conceituais de distintos domínios disciplinares ao núcleo das Políticas, planejamento e gestão em saúde ${ }^{8}$. Assim, ainda que a Epidemiologia predomine, o núcleo das Ciências $\mathrm{Hu}$ manas e Sociais vem fortalecendo as bases epistemológicas do campo e gerando desdobramentos operacionais mais consistentes, tanto no âmbito das práticas quanto nos locais de formação e de geração de conhecimentos em saúde ${ }^{14}$. Contudo, cabe salientar que a incorporação das Ciências Humanas e Sociais ainda não ocorreu em sua plenitude, havendo espaço para considerações mais refinadas da subjetividade produzida e produtora da práxis em saúde, projeto que vem ganhando força na produção mais recente ${ }^{15}$.

Por fim, o núcleo das Políticas, planejamento e gestão em saúde incorpora elementos conceituais das Ciências Humanas e Sociais à dimensão política, enfatizando questões vinculadas às relações de poder, bem como àquelas referidas no planejamento e no estabelecimento de diretrizes, planos, programas e ações em âmbito populacional, e suas implementações e avaliações ${ }^{16}$.

A SC como campo científico assumidamente interdisciplinar, atrai profissionais de diversas origens e incursiona por outros campos científicos mediante seus três núcleos fundamentais de saberes $^{8}$. Isso se torna evidente nas últimas duas décadas, quando a SC passa a refletir sobre e se ocupar da promoção da saúde através da cultura corporal do movimento humano e, para tal, se aproxima da $\mathrm{EF}^{11}$. No entanto, antes de analisarmos as interfaces entre ambos, cabe recuperarmos as origens, a trajetória e a constituição do campo da EF.

\section{Educação Física como campo de saberes e práticas}

O campo da EF brasileira se constitui apoiado no modo de produção capitalista da primeira metade do século XX, quando a prática sistematizada de movimentos físicos estava a cargo das instituições militares. Afiliado aos pensamentos nacionalista, militarista e biomédico, buscava "educar" o corpo para a produção e o combate na perspectiva da aptidão física, da eugenia e da segurança nacional ${ }^{17}$.

Tentativas de avanço de teorias críticas da educação na EF foram abortadas pelo golpe militar de 1964 que importou o modelo americano de esporte competitivo para o Brasil, bastante apropriado à ocasião por defender o individualismo disciplinado, o autossacrifício e o devotamento ao país como causa comum ${ }^{18}$. A EF brasileira incorporou, assim, saberes e práticas que buscavam o aprimoramento físico e técnico do indivíduo apoiado nos pressupostos da racionalidade, produtividade e eficiência mecanicista ${ }^{19}$. É nesse contexto sociopolítico que surgem os primeiros cursos de pós-graduação em EF, centrados na vertente biológica, fortemente marcados pela fisiologia do exercício e pela cinesiologia, e sem relação com o contexto social, pedagógico ou sanitário ${ }^{5}$.

A partir de 1980, refletindo o processo político de redemocratização do país, num movimento epistemológico próximo ao da SC, a EF incorpora efetivamente disciplinas relacionadas às Ciências Humanas e Sociais, e passa a realizar reflexões críticas sistemáticas em busca de seus propósitos voltados à educação e à sociedade, fundamentadas principalmente no materialismo dialético e respaldadas pela concepção históricocrítica da Filosofia da Educação ${ }^{17,20}$. Ao mesmo tempo, as academias, suas diferentes modalidades de exercícios e a visão da aptidão física (fitness) passam a ser amplamente divulgadas no Brasil como essenciais à saúde ${ }^{21}$, numa perspectiva biomédica, individual e de consumo. Esses dois movimentos, contraditórios, exacerbam os embates epistemológicos e as disputas político-ideológicas entre os saberes sociais e biológicos tanto no plano conceitual quanto na práxis do campo $^{22}$. No entanto, nesse período não se observam análises ou críticas sistematizadas questionando o paradigma hegemônico da aptidão física como sinônimo de saúde ${ }^{23}$.

Nos anos de 1990 - com a rápida ampliação e diversificação do mercado de trabalho, que passa a incluir: áreas de atuação referen- 
tes aos bens e serviços ${ }^{24}$; a expansão acadêmica, com abertura de inúmeros cursos de graduação e pós-graduação ${ }^{25}$; a reestruturação e a divisão dos cursos de formação em licenciatura e bacharelado $^{26}$; o reconhecimento da profissão como pertencente à área de saúde ${ }^{27}$ e a regulamentação da profissão ${ }^{28}$ - o campo da EF dá seguimento às discussões epistemológicas buscando definir seu estatuto científico. No entanto, encontra dificuldades em delimitar objetos, métodos e linguagens próprios, retardando sua consolidação no campo cientifico ${ }^{5,20}$. Nesse período, debates inaugurais sobre EF e saúde foram observados, quando algumas instituições de ensino superior buscaram dar identidade à formação do bacharel com ênfase na saúde. Entretanto, como aponta Carvalho $^{29}$, grande parte desses esforços careceu de um olhar ampliado sobre o conceito de saúde.

Sem concordância sobre sua identidade e, ainda menos acerca das modalidades de formação ${ }^{7}$, o campo da EF cresce e se estabelece marcado por uma miríade de significados, em que o uso de uma diversidade de conceitos e classificações amplia as dificuldades de entendimento a respeito das propostas de formação, intervenção e investigação do campo. Constata-se um intenso debate, no qual várias denominações, conceituações e classificações são propostas para se analisar o mesmo fenômeno, a partir de uma confusão entre os objetivos e a natureza do termo utiliza$\mathrm{do}^{20,22}$. Termos como exercício, esporte, atividade física, ginástica, cultura corporal, movimento humano, prática corporal, motricidade, educação física e outros ${ }^{30}$ circulam em espaços e discursos distintos, ora como sinônimos, ora marcados por algumas especificidades que os afastam, em um movimento permanente entre senso comum e conceitos científicos ${ }^{5,31}$.

Não sendo propósito deste texto aprofundar a reflexão epistemológica sobre esse movimento, é importante destacar a tensão entre estruturas que operam tanto no plano conceitual quanto na práxis do campo. Como exemplo, destacamos o termo atividade física, muito utilizado no cotidiano leigo e de práticas contemporâneas em saúde, e que mantém ligações intrínsecas com o paradigma biologizante da aptidão física e com o conceito de risco subjacente ao discurso corrente sobre o sedentarismo ${ }^{29}$. Em contrapartida, termos como práticas corporais e cultura corporal do movimento, dentre outros, buscam explicitar o caráter complexo do fenômeno e suas várias ligações sociais ${ }^{32}$. Num esforço analítico para sintetizar algumas convergências do campo, ainda que para isto tenhamos que atenuar diferenças e incorrer em simplificações, optamos por adotar, em consonância com o documento oficial das diretrizes curriculares nacionais para os cursos de graduação em $\mathrm{EF}^{30}$, que o objeto de estudo e prática da EF é o movimento humano, reconhecendo o caráter polissêmico do termo.

Entretanto, após “definir” o objeto, precisamos ainda identificar um núcleo básico que dê identidade ao "pensar" e ao "fazer" na EF"19. Seguindo o esforço de síntese a partir da multiplicidade de posições e formulações epistemológicas que existem, é possível identificar alguns pressupostos comuns que subjazem às proposições, permitindo distribuí-las em duas vertentes principais: a científica e a pedagógica ${ }^{22}$. Em linhas bastante gerais, a primeira se compõe de formulações - como a cinesiologia de Tani ${ }^{33}$; a ciência da motricidade humana de Manuel Sérgio ${ }^{34}$; as ciências do desporto de Gaya ${ }^{35}$; e a ciência do movimento humano de Canfield ${ }^{36}$ - que buscam a constituição de uma ciência autônoma substitutiva às abordagens disciplinares. Já a segunda se apoia em outro conjunto de saberes - tais como a prática pedagógica da EF como eixo integrador de Bratch $^{17}$; a EF como teoria da prática de Betti $^{37}$; o papel mediador da EF de Lovisolo ${ }^{38}$; e a EF como ciência da e para a prática interdisciplinar de Gamboa $^{39}$ - que refletem sobre a EF como uma prática pedagógico-social, com ênfase no seu papel educador mesmo na perspectiva científica.

A despeito de a fundamentação do campo científico da EF ocorrer na interface dos campos das Ciências Naturais com as Ciências Humanas e Sociais - lembrando a adjetivação "educação" que transpõe as Ciências Naturais - destacamos a posição hegemônica do modelo biomédico e a ausência de uma interdisciplinaridade no campo $^{40,41}$, explicitada quando a EF é inserida na denominada "grande área da Saúde" no sistema de avaliação da Coordenação de Aperfeiçoamento de Pessoal de Nível Superior (CAPES) ${ }^{20,25}$. Em consonância com outros cursos da área da saúde, a EF se configura predominantemente reduzida aos parâmetros da racionalidade científica moderna, derivados do método experimental e da tradição quantitativa de pesquisa, que deixam como legado a fragmentação e a neutralização de dimensões subjetivas fundamentais ao estudo da vida, da saúde, do corpo e de suas relações com o movimento humano ${ }^{42}$.

Tal concepção de ciência tem um óbvio impacto na produção e definição dos núcleos de saberes em EF. Apesar de, na teoria, haver um reconhecimento do caráter multidisciplinar - 
expresso na definição dos conteúdos curriculares da formação: relação ser humano-sociedade, biologia do corpo humano e produção do conhecimento científico e tecnológico ${ }^{30}$ - na realidade, a EF constrói sua base cognitiva de forma fragmentada, com hegemonia biodinâmica ${ }^{41}$. Empresta da Medicina conteúdos que permitem a análise do corpo e se aproxima da Educação para construir a essência de sua prática ${ }^{17,40}$.

Essa luta entre os campos da Educação e da Saúde (na visão hegemônica da aptidão física) pelos monopólios da autoridade e da competência científica se reflete também na atuação profissional em $\mathrm{EF}^{20}$. A despeito das inúmeras possibilidades de atuação - que contemplam o movimento humano em diferentes modalidades (exercício, ginástica, jogo, esporte, luta/arte marcial, dança) e perspectivas (prevenção, promoção, proteção e reabilitação da saúde; formação cultural; educação e reeducação motora; rendimento físico-esportivo; lazer; gestão de empreendimentos relacionados a estas temáticas; dentre outros) $)^{30}-$ os campos clássicos de intervenção profissional, que recebem quase a totalidade dos egressos que atuam na EF, são escolas e academias ${ }^{43}$.

O tensionamento culmina com a divisão dos cursos em licenciatura e bacharelado ${ }^{26}$, embora até recentemente a Resolução CFE 03/1987 não havia ocasionado resultados práticos quanto à diferenciação da formação. A questão ideológica do debate é a característica eminentemente pedagógica da prática em EF, a despeito do lócus de atuação. Politicamente, o Conselho Federal de EF (CONFEF) tenta garantir a atuação exclusiva dos bacharéis nos espaços não escolares já que o Ministério da Educação exige a formação em licenciatura para atuação nas escolas.

Não obstante os embates entre biológico e social, saúde e educação, bacharelado e licenciatura (tanto no plano conceitual quanto na práxis) destacamos que, no sentido mais geral e difundido, o campo da EF sempre buscou promover a aptidão física e a saúde expressas pela capacidade de trabalho e/ou de rendimento individual, adotando primordialmente a concepção anátomo-fisiológica de corpo a ser desenvolvido numa perspectiva técnico-desportiva ${ }^{17,40}$. Assim, a transdisciplinaridade exigida pelo objeto de estudo e prática da EF continua reduzida à dualidade fragmentada, que cria modelos ideais fechados para fenômenos sociais complexos que se manifestam dinamicamente nos espaços sociais, gerando desafios e impasses teóricos e práticos e dificultando o avanço do campo ${ }^{39,41}$.
No século XXI, as novas relações e organizações sociais (tal como a globalização); as mudanças sociossanitárias decorrentes dos aumentos da expectativa de vida, do excesso de peso e da prevalência de doenças crônicas; e as transformações nas relações do homem com seu corpo em movimento no lazer, no trabalho e no deslocamento, tornam imprescindível repensar as necessidades humanas e valorizam a atividade física como elemento chave na promoção da saúde ${ }^{1}$. Esse contexto favorece o encontro dos campos científicos da EF com a SC no Brasil, ainda que muito baseado na perspectiva do risco ${ }^{44}$.

A publicação da Política Nacional de Promoção da Saúde ${ }^{1}$, que institucionaliza no nível Federal as atividades físicas/práticas corporais como ações prioritárias de promoção da saúde, e seus desdobramentos - a criação dos Núcleos de Apoio à Estratégia Saúde da Família com oportunidade para o profissional de $\mathrm{EF}^{2}$, a criação de Programas como o Academia da Saúde ${ }^{3}$ e a gradual inserção da EF no Pró-Saúde (Programa Nacional de Reorientação da Formação Profissional em Saúde) $)^{45}$ e no PET-Saúde (Programa de Educação pelo Trabalho para a Saúde $)^{46}$ - passam a demandar uma nova dinâmica nos espaços de formação e no mercado de trabalho da EF, que agora inclui os serviços públicos de saúde. No início, a concepção de EF levada a interagir com a SC refletia o modelo de valorização da atividade física como fator de promoção da saúde (em sua perspectiva comportamentalista do controle de riscos), promovendo o melhor ajuste do homem à sociedade capitalista através de uma boa aptidão física ${ }^{31,44}$. Não obstante, é importante citar o processo de revisão da PNPS que ocorreu em 2014 com o intuito de superar ou ao menos minimizar o caráter epidemiológico, reprodutor da responsabilidade individual nos discursos e práticas governamentais em promoção da saúde ${ }^{47}$.

Conforme apresentaremos a seguir - apesar de ainda manter algumas características e tensões manifestas nos campos de origem, como a polarização entre as abordagens biológica e social - a constituição da EFSC promoveu um conjunto intrincado de movimentos epistemológicos e políticos que permeiam e modificam os campos de origem, abrindo possibilidades de reflexões em busca de paradigmas capazes de subsidiar projetos inovadores de movimento humano voltados para a saúde, em sentido complexo e multidimensional, tal como exigem os desafios contemporâneos à saúde e à vida ${ }^{44}$. 


\section{Educação Física em Saúde Coletiva: que campo é este?}

O arcabouço teórico-metodológico da Epidemiologia moderna é um dos primeiros fundamentos de vinculação entre a EF e a SC. Estudos inaugurais sobre a relação entre atividade física e doenças cardiovasculares desenvolvidos em Londres, comparando a taxa de mortalidade entre carteiros e trabalhadores de escritório do serviço postal e entre motoristas e cobradores dos ônibus de dois andares, mostraram que atividades ocupacionais com maior gasto energético estavam associadas com menor mortalidade por doenças coronarianas $^{48}$.

A partir da segunda metade do século XX no Brasil, as "transições epidemiológica, demográfica e nutricional" redirecionam significativamente o foco das pesquisas epidemiológicas para a obesidade e as doenças crônico-degenerativas, exigindo novos investimentos metodológicos e inserindo de vez o sedentarismo como fator determinante de agravos à saúde. Assim, o cenário de embates que marcam a Epidemiologia no campo da SC se replica no campo da EF. Hoje abundam estudos epidemiológicos que consistentemente demonstram a associação entre maiores níveis de atividade física e/ou aptidão física e a diminuição no risco de doenças crônicas ${ }^{49}$.

A "Epidemiologia da atividade física e saúde" transcorre fundamentada nas Ciências Biológicas, naturaliza a linguagem do risco e desconsidera a natureza multidimensional de fenômenos como a saúde e o movimento. A operacionalização do método ganha destaque em detrimento das reflexões teóricas acerca das relações humanas mediadas pelo movimento corporal, fixando-se no seu desfecho: a aptidão física. Ao adotar um âmbito mais técnico e se afastar das abordagens sociais com suas reflexões teóricas e epistemológicas, a "Epidemiologia da atividade física" contribui mais para a fragmentação de saberes e práticas em EF e Saúde do que para sua construção em fundamentos interdisciplinares ${ }^{40,41}$. Reproduz, mais uma vez, a força do método em detrimento do enunciado, uma das marcas da racionalidade científica moderna, se identificando fortemente com a economia dominante no campo científico ${ }^{42}$.

Esse processo, evidentemente, não se dá sem embates. O movimento de resistência ao modelo científico hegemônico da EF a partir das teorias críticas da Educação ${ }^{31}$ se fortalece ao se aproximar dos programas de pós-graduação em SC como loci privilegiados para atender às de- mandas por formação crítica $^{11}$. Ainda que em proporções minoritárias, o núcleo "Epidemiologia da atividade física" vai ampliando a visão de seus objetos de estudo mediante o emprego dos aportes da Epidemiologia Social, articulando informações sobre comportamentos e agravos ao contexto nacional de fortes iniquidades histórico-sociais, possibilitando novas frentes de estudo e atuação $0^{49}$.

O encontro com as teorias críticas da SC resulta ainda no aumento significativo de estudos e publicações sobre o caráter social e humano do movimento e da saúde ${ }^{44,50}$, incluindo estudos sobre a formação em EFSC ${ }^{51-54} \mathrm{e}$ análises e avaliações mais politizadas de programas e ações governamentais de combate ao sedentarismo e da atuação do profissional de EF na promoção da saúde ${ }^{23,55-58}$.

Assim, as reflexões abarcadas pelas Ciências Humanas e Sociais em EF e Saúde implicam na reorientação do que a EF reconhece como saúde. Há que se distinguir a aproximação inicial da EF com as Ciências Humanas e Sociais nos anos de 1980, que realizou reflexões embasadas nas teorias críticas da Educação, desta reaproximação atual desencadeada pelo contato com a SC. Esses referenciais, seminais na conformação do campo da SC, trouxeram ao da EF questionamentos acerca dos fundamentos e subjetividades implicadas com o movimento humano e de seu alcance para descrever e explicar questões como determinação social do sedentarismo, da saúde e de outros fenômenos que escapam aos modelos causais lineares, configurando, gradualmente, novos objetos de estudo e atuação $0^{50,53}$.

A reaproximação da EF com as Ciências Humanas e Sociais pela via da SC tem ainda maior relevância se a pensarmos contextualizada no momento pós-divisão da formação profissional em licenciatura e bacharelado quando, grosso modo, a primeira opção de formação manteria o legado das reflexões pedagógicas de cunho $\mathrm{Hu}$ manístico e a segunda estaria fadada quase que exclusivamente às Ciências Biológicas, não fosse o encontro com a SC ${ }^{40,41,59}$. Entretanto, esta perspectiva não indica que o desenvolvimento da EFSC se aproxime apenas do conjunto de saberes e práticas do bacharelado. Pelo contrário, o caráter transdisciplinar da SC vem sendo fortalecido a partir de propostas intersetoriais como, por exemplo, o Programa Saúde na Escola ${ }^{60}$ e o Pró/ PET-Saúde, programas desenvolvidos em parceria pelos Ministérios da Saúde e da Educação ${ }^{45,46}$.

Embora existam registros de produções científicas com enfoque social que reconhecem a na- 
tureza multidimensional e holística da relação homem-movimento-sociedade-saúde no campo da EF desde meados de $1990^{29}$, o aumento desse tipo de produção é indiscutível. Hoje contamos com reflexões que permitem "pensar" a EF e a saúde como possibilidades de realização humana, incluindo o lugar dos atores sociais em suas relações coletivas; a compreensão e a inclusão das subjetividades como fenômenos situados no seu contexto histórico; a análise crítica no estudo de intervenções e na formação de profissionais; e a revisão da práxis e dos paradigmas dominantes no mundo da ciência, dentre tantos outros ${ }^{23,44,50,51,53,54,56-59}$.

Quanto a análises histórico-críticas de políticas e programas sociais, podemos afirmar que essas são mais comuns ao campo da EF partindo de olhares sobre o Esporte, o Lazer e a Educação $\mathrm{o}^{20,61}$. No entanto, análises voltadas a apontar os limites e as contradições políticas da EFSC são mais escassas. A materialização desse terceiro núcleo de saberes - "Política, planejamento e gestão em EF e Saúde" - vem sendo possível apenas na última década, quando as atividades físicas/práticas corporais são assumidas como prioridade no complexo conjunto de políticas, estratégias e programas de combate às doenças crônico-degenerativas e de promoção da saúde formuladas no âmbito do Estado brasileiro ${ }^{1-3,45-47,60}$

Acompanhando o aumento recente de programas e financiamentos relativos à atividade física e saúde, alguns trabalhos vêm tentando dar maior visibilidade ao pensamento crítico, denunciando o caráter intrinsecamente epidemiológico, reprodutor do capital e da responsabilidade individual nos discursos e práticas governamentais formuladas a partir de estudos que defendem a relação entre atividade física e promoção da saúde ${ }^{47,55,62-64}$.

Não obstante esses avanços, gostaríamos de destacar alguns aspectos que ainda consideramos desafios ao campo da EFSC. Primeiro, apontamos fragilidades e inconsistências epistemológicas e a necessidade de maior embasamento teórico-metodológico em algumas das produções contemporâneas. Se por um lado, a "atividade física” ganha lugar na SC, esta, mesmo que de forma disfarçada, mantém o espaço dos referenciais biomédicos e normalizadores. Por outro lado, a inserção de termos como "práticas corporais" tenta trazê-la para dentro das perspectivas socioculturais, destacando assim suas distinções ${ }^{31,41,44}$. No entanto, muitos pesquisadores desconsideram esse tensionamento em suas análises.
Segundo, os desdobramentos operacionais, ou seja, as repercussões desses estudos nas práticas em saúde e nos locais de formação ainda são tímidos ${ }^{59}$. A concretude das ações da EFSC, tanto no âmbito da formulação quanto da execução, ainda evidencia a "crise epistemológica e de identidade" dos campos originais ${ }^{23}$. O terceiro desafio se refere à construção interna da interdisciplinaridade na EFSC. A despeito da aproximação entre os três núcleos de saber - Epidemiologia, Ciências Humanas e Sociais e Políticas na EFSC - essa dinâmica simultaneamente explicita e aprofunda as tensões conceituais que busca enfrentar. A exigência de foco nas ações e resultados concretos e imediatos no âmbito das "Políticas em EFSC" pode afastar os atores da necessária reflexão conceitual acerca dos paradigmas em disputa.

\section{Considerações finais}

Com o intuito de contribuir com a reflexão sobre o que caracteriza e demarca a EFSC o presente artigo apresentou alguns elementos desse novo objeto de reflexão a partir dos campos de origem. Conforme buscamos evidenciar, há um movimento marcado pelo encontro epistêmico entre as configurações constitutivas do campo da SC e uma parcela do campo da EF orientado por núcleos de saberes diferenciados dos demais, dando origem a um específico de saberes e práticas distintas, que denominamos EFSC. Esse processo é marcado por tensões entre os paradigmas biológico e social, se configurando como um importante desafio a ser enfrentado.

O uso dos referenciais das Ciências Humanas e Sociais ainda é limitado nos estudos, tanto no que concerne a abordagens macrossociológicas como aquelas que tratam da microfísica na qual se tecem as relações cotidianas de poder no setor saúde. No entanto, as iniciativas, as discussões e as intervenções que germinam na EFSC estão sendo profundamente reconfiguradas mediante a reconstrução de conceitos através do referencial crítico adotado no pensamento social em saúde, um movimento crescente com o maior trânsito de profissionais de EF no campo da SC, operando uma busca permanente de superação dialética nos saberes e nas práticas da EFSC. A incorporação da categoria no SUS é recente, se comparada a outras profissões, objeto que merece aprofundamento em outras análises, mas extrapola os objetivos deste artigo. 


\section{Colaboradores}

JAD Nogueira e MLM Bosi participaram de todas as etapas da produção do presente artigo, da concepção e desenvolvimento à revisão final, assumindo a responsabilidade pública pelo seu conteúdo.

\section{Referências}

1. Brasil. Ministério da Saúde (MS). Política Nacional de Promoção da Saúde. Brasília: MS; 2006.

2. Brasil. Ministério da Saúde (MS). Portaria no 154, de 24 de janeiro de 2008. Cria os Núcleos de Apoio à Saúde da Família. Diário Oficial da União 2008; 24 jan.

3. Brasil. Ministério da Saúde (MS). Portaria no 719, de 7 de abril de 2011. Institui o Programa Academia da Saúde no âmbito do Sistema Único de Saúde. Diário Oficial da União 2011; 7 abr.

4. Nunes ED. Saúde Coletiva: história recente, passado antigo. In: Campos GWS, Carvalho YM, Minayo MCS, Drumond Junior M, Akerman M. organizadores. Tratado de saúde coletiva. $2^{\text {a }}$ ed. São Paulo, Rio de Janeiro: Hucitec, Fiocruz; 2009. p. 19-40.

5. Bracht VA. Educação Física e Ciência: cenas de um casamento (in) feliz. Rev. Bras. Cienc. Esporte 2000; 22(1):53-65.

6. Paim JS, Bosi MLM. Graduação em saúde coletiva: limites e possibilidades como estratégia de formação profissional. Cien Saude Colet 2010; 15(4):2029-2038.

7. Souza Neto S, Nardi Alegre A, Hunger D, Pereira JM. A formação do profissional de Educação Física no Brasil: uma história sob a perspectiva da legislação federal no século XX. Rev. Bras. Cienc. Esporte 2004; 25(2):113128.

8. Bosi MLM, Prado SD. Alimentação e Nutrição em Saúde Coletiva: constituição, contornos e estatuto científico. Cien Saude Colet 2011; 16(1):7-17.

9. Khun T. A estrutura das revoluções científicas. São Paulo: Perspectiva; 2003.

10. Bourdieu P. O campo científico. In: Ortiz R. organizador. Pierre Bourdieu: Sociologia. São Paulo: Ática; 1983. p. 122-155.

11. Campos GWS, Carvalho YM, Minayo MCS, Drumond Junior M, Akerman M. organizadores. Tratado de Saúde Coletiva. São Paulo, Rio de Janeiro: Hucitec, Fiocruz; 2009.

12. Czeresnia D. Epidemiologia, Ciências Sociais, integração das ciências. Rev Saude Publica 2008; 42(6):11121117.

13. Paim JS. Desafios para a Saúde Coletiva no século XXI. Salvador: EdUFBA; 2006.

14. Loyola MAR. A saga das Ciências Sociais na área da Saúde Coletiva: elementos para reflexão. Physis 2008; 18(2):251-275.

15. Mercado FJ, Bosi MLM. Notas para um debate In: Bosi MLM, Mercado FJM. Pesquisa qualitativa de serviços de saúde. Petrópolis: Vozes; 2006. p. 23-71.

16. Paim JS, Teixeira CF. Política, planejamento e gestão em saúde: balanço do estado da arte. Rev Saude Publica 2006; 40(n. esp):73-78

17. Bracht VA. Constituição das teorias pedagógicas da educação física. Cad Cedes 1999; 19(48):69-88.

18. Castelani Filho L. Educação Física no Brasil: a história que não se conta. Campinas: Papirus; 1988.

19. Melo VA. Relação teoria \& prática e formação profissional na Educação Física brasileira: apontamentos na história. Motrivivência 1996; 7(8):103-115.

20. Lazzarotti Filho A, Silva AM, Marcarenhas F. Transformações contemporâneas do campo acadêmico-científico da educação física no brasil: novos habitus, modus operandi e objetos de disputa. Movimento 2014; 20 (n. esp):67-80. 
21. DaCosta L, organizador. Atlas do esporte no Brasil. Rio de Janeiro: CONFEF; 2006.

22. Lima HLA. Pensamento epistemológico da educação física brasileira: das controvérsias acerca do estatuto científico. Rev. Bras. Cienc. Esporte 2000; 21(2/3):95102.

23. Bagrichevsky M, Estevão A, Palma A. La Educación Física y su inserción en el campo de la Salud Colectiva en Brasil: en búsqueda de un diálogo crítico sobre interfaces, pertenencias y sentidos. Physis 2008; 18(1):123142.

24. Taffarel CNZ. Currículo, formação profissional na educação física \& esporte e campos de trabalho em expansão: antagonismos e contradições da prática social. Movimento 1997; 4(7):43-51.

25. Rigo LC, Ribeiro GM, Hallal PC. Unidade na diversidade: desafios para a Educação Física no século XXI. Rev bras. ativ. fís. saúde 2011; 16(4):339-345.

26. Brasil. Ministério da Educação. Conselho Federal de Educação. Resolução CFE no 3, de 16 de julho de 1987. Fixa os mínimos de conteúdo e duração a serem observados nos cursos de graduação em Educação Física (Bacharelado e/ou Licenciatura Plena). Diário Oficial da União 1987; 16 jul.

27. Brasil. Ministério da Saúde (MS). Conselho Nacional de Saúde. Resolução no 218, de 6 de Março de 1997. Reconhece profissões da área da saúde. Diário Oficial da União 1997; 6 mar.

28. Brasil. Casa Civil. Lei $\mathrm{n}^{\circ}$ 9696, de 1 de setembro de 1998. Dispõe sobre a regulamentação da profissão de educação física e cria os respectivos conselhos Federal e Conselhos Regionais de educação física. Diário Oficial da União 1998; 1 set.

29. Carvalho YM. O mito atividade física/saúde [dissertacão]. Campinas: Faculdade de Educação Física; 1993.

30. Brasil. Ministério da Educação. Conselho Nacional de Educação. Câmara de Educação Superior. Resolução CNE/CES no 7, de 31 de março de 2004. Institui as Diretrizes Curriculares Nacionais para os cursos de graduação em Educação Física, em nível superior de graduação plena. Diário Oficial da União 2004; 31 mar.

31. Betti M, Knijnik J, Venâncio L, Neto LS, Daolio J. Fundamentos filosóficos e antropológicos da Teoria do Se-movimentar e a formação de sujeitos emancipados, autônomos e críticos: o exemplo do currículo de Educação Física do Estado de São Paulo. Movimento 2014; 20(4):1631-1653.

32. Lazzarotti Filho A, Silva AM, Cesaro Antunes P, Silva APS, Leite JO. O termo práticas corporais na literatura científica brasileira e sua repercussão no campo da Educação Física. Movimento 2010; 16(1):11-29.

33. Tani GO. Cinesiologia, educação física e esporte: ordem emanente do caos na estrutura acadêmica. Motus corporis 1996; 3(2):9-50.

34. Sergio M. Motricidade humana: um paradigma emergente. Blumenau: FURB; 1995.

35. Gaya AC. As ciências do desporto no espaço de língua portuguesa. Porto: Universidade do Porto; 1994.

36. Canfield JT. A ciência do movimento humano como área de concentração de um programa de pós-graduação. Rev. Bras. Cienc. Esporte 1993; 14(3):46-148.

37. Betti M. Por uma teoria da prática. Motus corporis 1996; 3(2):73-127.
38. Lovisolo H. Educação Física: a arte da mediação. Rio de Janeiro: Sprint; 1995.

39. Gamboa SS. Pesquisa em educação física: as inter-relações necessárias. Motrivivência 1994; 1(5):34-46.

40. Betti M, Carvalho YMD, Daolio J, Pires GDL. A avaliação da educação física em debate: implicações para a subárea pedagógica e sociocultural. RBPG 2011; 1(2):183-194.

41. Manoel EJ, Carvalho YM. Pós-graduação na educação física brasileira: a atração (fatal) para a biodinâmica. Educação e Pesquisa (SP) 2011; 37(2):389-406.

42. Luz MT. Natural, racional, social: razão médica e racionalidade moderna. São Paulo: Hucitec; 2004.

43. Dantas ER. Os campos de atuação profissional da educação física: um olhar sobre o mercado de trabalho no interior da Paraíba. Holos 2009; 1:24-30.

44. Luz MT. Novos saberes e prática em saúde coletiva: estudo sobre racionalidades médicas e atividades corporais. São Paulo; Hucitec; 2005.

45. Brasil. Ministério da Saúde (MS). Ministério da Educação. Portaria Interministerial no 3.019, de 26 de novembro de 2007. Dispõe sobre o Programa Nacional de Reorientação da Formação Profissional em Saúde (Pró-Saúde) para os cursos de graduação da área da saúde. Diário Oficial da União 2007; 26 nov.

46. Brasil. Ministério da Saúde (MS). Ministério da Educação. Portaria Interministerial no 1.802 , de 26 de agosto de 2008. Institui o Programa de Educação pelo Trabalho para a Saúde (PET-Saúde). Diário Oficial da União $2008 ; 26$ ago

47. Rocha DG, Alexandre VP, Marcelo VC, Rezende R, Nogueira JAD, Sá RF. Processo de revisão da Política Nacional de Promoção da Saúde: múltiplos movimentos simultâneos. Cien Saude Colet 2014; 19(11):4313-4322.

48. Morris JN, Heady JA, Raffle PAB, Roberts CG, Parks JW. Coronary heart disease and physical activity of work. I. Coronary heart disease in different occupations. Lancet 1953; 265(6795):1053-1057.

49. Hallal PC, Dumith SC, Bastos JP, Reichert FF, Siqueira FV, Azevedo MR. Evolução da pesquisa epidemiológica em atividade física no Brasil: revisão sistemática. Rev Saude Publica 2007; 41(3):453-460.

50. Alves FS, Carvalho YM. Práticas corporais e grande saúde: um encontro possível. Movimento 2010; 16(4):229-244

51. Bennedetti TRB, Silva DAS, Silva KS, Nascimento JV, organizadores. A formação do profissional de Educação Física para o setor saúde. Florianópolis: Postmix; 2014.

52. Guarda FRBD, Silva RND, Araújo Júnior JLDACD, Freitas MIDF, Neto S. Intervenção do profissional de educação física: formação, perfil e competências para atuar no Programa Academia da Saúde. Rev Pan-Amaz Saúde 2014; 5(4):63-74.

53. Carvalho YM, Rovigatti PA, Trevizan AA. Formação em Educação Física no Brasil: outros modos de pensar e intervir no serviço público de saúde. Educ Fís Cienc 2013; 15(1):1-6

54. Fraga $\mathrm{AB}$, Wachs $\mathrm{F}$, organizadores. Educação física $e$ saúde coletiva: políticas de formação e perspectivas de intervenção. Porto Alegre: UFRGS; 2007. 
55. Santos S, Benedetti TRB. Cenário de implantação do Núcleo de Apoio à Saúde da Família e a inserção do profissional de Educação Física. Rev. bras. ativ. fís. saúde 2013; 17(3):188-194.

56. Bagrichevsky M, Estevão A, Palma A, organizadores. A saúde em debate na Educação Física. Blumenau: Edibes; 2003.

57. Bagrichevsky M, Palma A, Estevão A, organizadores. A saúde em debate na Educação Física: volume 2. Blumenau: Nova Letra; 2006.

58. Bagrichevsky M, Estevão A, Palma A, organizadores. A saúde em debate na Educação Física: volume 3. Ilheus: Editus; 2007.

59. Anjos TC, Duarte ACGO. A Educação Física e a estratégia de saúde da família: formação e atuação profissional. Physis 2009; 19(4):1127-1144.

60. Brasil. Ministério da Saúde (MS). Ministério da Educação. Decreto no 6.286, de 5 de dezembro de 2007. Institui o Programa Saúde na Escola - PSE, e dá outras providencias. Diário Oficial da União 2007; 5 dez.

61. Programas sociais de esporte e lazer [editorial]. Motrivivencia 2012; 38:1-278.

62. Brasil. Ministério da Saúde (MS). Portaria Ministerial $\mathrm{n}^{\circ} 2.446$, de 13 de novembro de 2014. Redefine a Política Nacional de Promoção de Saúde. Diário Oficial da União 2014; 13 nov.

63. Scabar TG, Pelicioni AF, Pelicioni MCF. Atuação do profissional de Educação Física no Sistema Único de Saúde: uma análise a partir da Política Nacional de Promoção da Saúde e das Diretrizes do Núcleo de Apoio à Saúde da Família - NASF. J Health Sci Inst 2012; 30(4):411-418.

64. Cruz DKA. Da promoção à prevenção: o processo de formulação da política nacional de promoção da saúde no período de 2003 a 2006 [dissertação]. Recife: Fundação Oswaldo Cruz; 2010.

Artigo apresentado em 23/11/2015

Aprovado em 16/05/2016

Versão final apresentada em 18/05/2016 